\title{
DAILY SCHEDULING OF SMALL HYDRO POWER PLANTS DISPATCH WITH MODIFIED PARTICLES SWARM OPTIMIZATION
}

\author{
Sinvaldo Rodrigues Moreno ${ }^{1 *}$ and Eloy Kaviski ${ }^{2}$
}

Received January 26, 2012 / Accepted December 2, 2013

\begin{abstract}
This paper presents a new approach for short-term hydro power scheduling of reservoirs using an algorithm-based Particle Swarm Optimization (PSO). PSO is a population-based algorithm designed to find good solutions to optimization problems, its characteristics have encouraged its adoption to tackle a variety of problems in different fields. In this paper the authors consider an optimization problem related to a daily scheduling of small hydro power dispatch. The goal is construct a feasible solution that maximize the cascade electricity production, following the environmental constraints and water balance. The paper proposes an improved Particle Swarm Optimization (PSO) algorithm, which takes advantage of simplicity and facility of implementation. The algorithm was successfully applied to the optimization of the daily schedule strategies of small hydro power plants, considering maximum water utilization and all constraints related to simultaneous water uses. Extensive computational tests and comparisons with other heuristics methods showed the effectiveness of the proposed approach.
\end{abstract}

Keywords: particle swarm optimization, small hydro power plants, daily scheduling.

\section{INTRODUCTION}

In last ten years, a new type of hydro power plant called Small Hydro Power Plant (SHPP) has been introduced into the Brazilian Power System. Small Hydro Power Plants have been identified as one of the most important energy sources that can provide convenient and uninterrupted energy to remote rural communities, small cities or industries. Hydro power plant is typically defined as SHPP for an installed capacity less than $30,000 \mathrm{~kW}$ and small reservoir with less than $3 \mathrm{~km}^{2}$ dedicated water surface area (ANEEL, 2003). SHPP are a renewable source of energy which can still be strongly developed all over the world. However, due to increasingly growing conflicts among competing objectives, daily SHPP dispatch became highly complex and more dynamic.

\footnotetext{
*Corresponding author.

${ }^{1}$ University Federal of Paraná - UFPR. E-mail: sinvaldo.moreno@ufpr.br

${ }^{2}$ Department of Hydraulics - PPGERHA - UFPR, P.O BOX 19011, Jd. Americas, 81351-990 Curitiba, PR, Brazil. E-mail: eloy.dhs@ufpr.br
} 
Important constraints underlying the characteristics of SHPP dispatch in addition to the traditional objectives of water supply, including water quality, endangered species habitat preservation, and various recreational uses must be considered priority on operational hydro power production model. Nevertheless for the operation of SHPP, simple and generally applicable daily power dispatch optimization procedures are lacking. Among the few works found related to SHPP, Anagnostopoulos \& Papantonis (2007) presented a Stochastic Evolutionary Algorithm for the optimal sizing of a small hydro power plant that simulates in detail the plant operation during the year with the aim of maximizing the economic benefit and the energy produced. Peña et al (2009) estimated the capacity of a SHPP based on time series forecasting.

Acosta et al. (2012) had explored the applicability of the distributed generation concept in a small city with renewable energy provided by biomass and small hydro power plants in a microgrid configuration. The management of the generators is performed with in an economic dispatch framework with modified metaheuristic optimization algorithms. The main concern of the work is find the most profitable dispatch configuration in a deregulated market. Varfolomejeva et al. (2013) had applied dynamic programming (DP) and generalized reduced gradient method (GRG) to a single small hydro power plant (SHPP). The authors considered the operating regime optimization from the point of view of increasing the income during its whole day work. The obtained results show that the GRG method can provide more accurate result than DP method. The main conclusion is because GRG method does not depend on the reservoir water level discretization.

The hydro power problem examined in this paper is inherently nonlinear with both nonlinear and linear constraints. Both traditional and meta heuristic algorithms can be applied to these type of problems. Very fast and incredibly reliable traditional algorithms are available for solving problems with linear objective functions and constraints. However, traditional algorithms are typically less efficient when applied to nonlinear objectives and nonlinear constraints. They typically require longer solutions time and can fail to identify a solution more frequently in this setting. Recently, a new optimization method called the Particle Swarm Optimization (PSO) was designed to find good solutions to optimization problems. This algorithm is a population-based algorithm and was introduced by Kennedy \& Eberhart (1995). The technique was inspired by bird flocking and animal social behaviors. The main advantage of the PSO algorithm is that its computational cost is lower, and immediately implementable on personal computers as well as on more sophisticated machines. Its characteristics have encouraged its adoption to tackle a variety of problems in different fields.

This paper analyzes the results obtained by applying PSO algorithm to an optimization problem related to the Daily Hydroelectric Generation Scheduling (DHGS) of small hydro power plants. In order to improve the performance of standard PSO, this work attempts to introduce a modified particle swarm optimization algorithm, where the capacity of solving nonlinear problem is enhanced effectively through adjusting inertia factor dynamically, combined on local and global search methods of PSO algorithm. The main contribution of this paper is an alternative approach based on the fact that PSO techniques are designed to solve the daily small hydro power plants 
scheduling problem. This kind of power plant requires special approach due the hydrology seasonality and the small regulation reservoir capacity. The efficiency of the proposed method is demonstrated through comparison of performance with Standard PSO and other heuristic algorithms, such as Simulated Annealing.

The review of the PSO algorithm is described in the next section. Section 3 elaborates on the implementation of the new approach of PSO. Section 4 presents the problem formulation of small hydro power plants scheduling and the results for the case study. Finally, the main conclusions are summarized in Section 5.

\section{PARTICLE SWARM OPTIMIZATION ALGORITHM}

The PSO algorithm, originally proposed by Kennedy and Eberhart (1995), was based on the social behavior of organisms such as birds flocking and fish schooling, coupled with swarm theory. Generally, the main idea of PSO can be described as follows: firstly, a swarm of particles is randomly initialized with a position and velocity. Assuming the search space $S$ is $D$ dimensional, the position of the $i^{\text {th }}$ particle of a swarm can be represented by a $D$-dimensional vector $\vec{X}_{i}=\left[x_{i 1}, x_{i 2}, \cdots, x_{i D}\right]^{T}$ where each particle moves in $S$ with an adaptable velocity $\vec{V}_{i}=\left[v_{i 1}, v_{i 2}, \cdots, v_{i D}\right]^{T}$. This way, the best position the $i^{\text {th }}$ particle has ever visited in the search space is recorded and represented as $\vec{P}_{i}=\left[p_{i 1}, p_{i 2}, \cdots, p_{i D}\right]^{T}$. The best position of the best particle in the swarm is denoted by $P_{\text {best }}^{k}$, therefore, the new velocity and position of the $i^{\text {th }}$ particle is adjusted as follows:

$$
\begin{gathered}
\vec{V}_{i}^{k+1}=\omega \vec{V}_{i}^{k}+c_{1} r_{1}^{k}\left(\vec{P}_{i}^{k}-\vec{X}_{i}^{k}\right)+c_{2} r_{2}^{k}\left(P_{\text {best }}^{k}-\vec{X}_{i}^{k}\right) \\
\vec{X}_{i}^{k+1}=\vec{X}_{i}^{k}+\vec{V}_{i}^{k+1}
\end{gathered}
$$

where $i=1,2, \ldots, n ; n$ is total of particles in the swarm; $k=1,2, \ldots, k^{\text {th }}$ is the iteration counter; $r_{1}$ and $r_{2}$ are two random numbers uniformly distributed in $[0,1] ; c_{1}$ and $c_{2}$ are two positive constants called individual cognitive level and social cognitive level, respectively. Both $c_{1}$ and $c_{2}$ are generally 2.05; and $\omega$ is the inertial weight coefficient, which controls the influence of velocity of the previous iterations on the current iteration.

Each particle is considered to have a neighborhood that consists of a number of other particles and its movement is influenced by their experience (i.e., best positions). The neighborhoods can be defined in different ways. Different topologies have been proposed and applied with promising results by Kennedy (2003) and by Mendes et al. (2004). The most common neighborhood topology is the ring topology, where the immediate neighbors of the particle $x_{i}$ are the particles $x_{i-1}, x_{i+1}$ and $x_{i}$ is considered to be the particle that follows immediately after $x_{n}$. The ring topology is the scheme adopted in the current study. All vector operations in equations (1) and (2) are performed to each and every component of the matrix. A stability analysis of PSO, as well as recommendations regarding the selection of its parameters are provided by Clerc \& Kennedy (2002) and Trelea (2003). 


\subsection{Inertial Weight Coefficient}

A suitable selection of the inertial weight coefficient has great influence in the performance of the PSO algorithm. A larger inertial weight coefficient $\omega$ improves the convergence speed of global exploration while a smaller inertial weight coefficient tends to facilitate local exploration to the current search area. In general, a linearly decreasing inertial weight coefficient that was proposed by Shi \& Eberhart (1998a, 1998b) is used in standard PSO. The procedure represented in equation (3), $w$ often is decreased linearly from about 0.9 to 0.4 during a run, $k^{\text {th }}$ represents the maximum allowed number of iterations and $k$ is the current iteration counter.

$$
\omega(k)=\omega_{\max }-\left(\frac{\omega_{\max }-\omega_{\min }}{k^{\text {th }}}\right) k
$$

\section{MODIFIED PARTICLE SWARM OPTIMIZATION ALGORITHM}

In order to overcome the disadvantages of the conventional PSO, including prematurity, slow convergence, and liability to getting trapped in local optima, a new approach for PSO Algorithm is introduced in this paper. The improvement unifies the PSO Algorithm and a new scheme of linearly decreasing inertial weight coefficient $\omega$.

This approach shows similarity with Unified PSO algorithm (UPSO), developed by Parsopoulos \& Vrahatis (2004), but the main difference is the impact caused by the inertial weight coefficient $\omega$ associated to the constriction coefficient $\chi$ on the convergence behavior. This approach, called Modified UPSO (M-UPSO), is presented below.

\subsection{Unified Particle Swarm Optimization Algorithm}

Parsopoulos \& Vrahatis (2004) developed the UPSO approach as an algorithm that harnesses the global and local PSO variant in unified scheme, which combines their exploration and exploitation capabilities. These two variants of PSO are distinguished due to the number of particles that comprise the neighborhood of a particle. On the $L_{\text {best }}$ variant, known as local PSO variant, this number is related to the best particle in the neighborhood of $x_{i}$. On the $G_{b e s t}$, or global PSO variant, the number of particles are related to the best particle in the entire swarm. The following model uses a single constriction coefficient $\chi$, which was distinguished and studied further by Clerc \& Kennedy (2002).

Let $\vec{G}_{i}^{k+1}$ denote the velocity update of the particle $\vec{X}_{i}$ in the global PSO variant and let $\vec{L}_{i}^{k+1}$ denote the corresponding velocity update for the local variant, then:

$$
\begin{aligned}
& \vec{G}_{i}^{k+1}=\chi\left[\vec{V}_{i}^{k}+c_{1} r_{1}^{k}\left(\vec{P}_{i}^{k}-\vec{X}_{i}^{k}\right)+c_{2} r_{2}^{k}\left(G_{\text {best }}^{k}-\vec{X}_{i}^{k}\right)\right] \\
& \vec{L}_{i}^{k+1}=\chi\left[\vec{V}_{i}^{k}+c_{1} r_{1}^{k}\left(\vec{P}_{i}^{k}-\vec{X}_{i}^{k}\right)+c_{2} r_{2}^{k}\left(L_{\text {best }}^{k}-\vec{X}_{i}^{k}\right)\right] .
\end{aligned}
$$


These two search directions are combined in a single equation, resulting in the main UPSO scheme proposed by Parsapoulos \& Vhariats (2004):

$$
\begin{gathered}
\vec{V}_{i}^{k+1}=u \vec{G}_{i}^{k+1}+(1-u) \vec{L}_{i}^{k+1} \\
\vec{X}_{i}^{k+1}=\vec{X}_{i}^{k}+\vec{V}_{i}^{k+1}
\end{gathered}
$$

where $u \in[0,1]$ is a new parameter, called unification factor, which controls the influence of the global and local velocity update. $\chi$ is the constriction factor that normally assumes standard value 0.729 . The other parameters are the same as for the standard PSO, except for the inertial weight coefficient $w$ that was not longer used in UPSO scheme (Parsopoulos \& Vrahatis, 2004). The original global and local PSO variants constitute special cases of UPSO for $u=1$ and $u=0$, respectively. For all intermediate values $u \in[0,1]$ define UPSO variants that combine the exploration and exploitation properties of $G_{\text {best }}$ and $L_{\text {best }}$.

\subsection{Modified UPSO (M-UPSO)}

The PSO approach proposed by Shi \& Eberhart (1998a, 1998b) had introduce a concept of inertial weight. The motivation was to be able to eliminate the need of maximum velocity factor $V_{\max }$. As originally developed, $w$ often is decreased linearly from about 0.9 to 0.4 during a run. After some experience with the inertial weight, it was found that although the maximum velocity factor $V_{\max }$ could not be eliminated. However, the PSO algorithm still gets trapped in local optima.

A slight adjustment was made to the inertial weight to avoid the local optima. The solution proposed in this paper consists in refresh the inertial weight at end of each particle evaluation, assign to $\omega=\omega_{\max }$. This procedure is defined as:

$$
\omega(i)=\omega_{\max }-\left(\frac{\omega_{\max }-\omega_{\min }}{N}\right) i
$$

where $i$ is the current particle evaluated in current iteration and $N$ represents the total of particles in swarm. After applying the new inertial weight decrease procedure and the constriction coefficient $\chi$ to equations (4) and (5), the Modified UPSO scheme is derived as:

$$
\begin{gathered}
\vec{G}_{i}^{k+1}=\omega \vec{V}_{i}^{k}+\chi\left[c_{1} r_{1}^{k}\left(\vec{P}_{i}^{k}-\vec{X}_{i}^{k}\right)+c_{2} r_{2}^{k}\left(G_{\text {best }}^{k}-\vec{X}_{i}^{k}\right)\right] \\
\vec{L}_{i}^{k+1}=\omega \vec{V}_{i}^{k}+\chi\left[c_{1} r_{1}^{k}\left(\vec{P}_{i}^{k}-\vec{X}_{i}^{k}\right)+c_{2} r_{2}^{k}\left(L_{\text {best }}^{k}-\vec{X}_{i}^{k}\right)\right] \\
\vec{V}_{i}^{k+1}=u \vec{G}_{i}^{k+1}+(1-u) \vec{L}_{i}^{k+1} \\
\vec{X}_{i}^{k+1}=\vec{X}_{i}^{k}+\vec{V}_{i}^{k+1} .
\end{gathered}
$$


According to equations (9) and (10), the new position shift of a particle in M-UPSO consists in weighted combination of the $\vec{G}_{\text {best }}$ and $\vec{L}_{\text {best }}$ position shifts. Each position has the influence of inertial weight and the constriction coefficient. The unification factor balances the influence of these two search directions.

\section{APPLICATION ON SHORT-TERM OPTIMAL OPERATION OF CASCADE SMALL HYDRO POWER PLANTS}

The coordination of hydro power plants' operation involves the scheduling of water release. According to the scheduling period, the hydro system operation problem can be divided into long-term hydro-scheduling and short-term hydro-scheduling problems. The long-term hydroscheduling problem involves the long-term forecasting of water availability and the scheduling of reservoir water release for a time interval that depends on the reservoir capacity. Typical longterm scheduling goes anywhere from 1 week to 1 year or several years. For hydro schemes with a capacity of impounding water over several seasons, the long-term problem involves meteorological and statistical analysis. The present study focus is on the short-term hydro-scheduling problem.

Short-term hydro-scheduling refers to time periods from one day to one week. It involves the hour-by-hour scheduling of all power plants generation on a hydro system to achieve the better benefit cost relation for the given time period. In an optimization model applied for short-term hydro-scheduling where the cascade reservoirs have small storage capability, the main objective is regulating water flows for a few hours and providing a flat daily hydro power production. When necessary, hydroelectric generation shifts its scheduling along the day to cover on demand peak load.

The present study aims to maximize the daily hydro power production. Thus the paper unifies PSO theory and small reservoir operation, the outflows from reservoir storage corresponding to particles, amount of scheduling period to dimension of searching space, generation objective function to fitness function. That way, one particle can be indicated as $m \times n$ matrix $\left(\mathbf{X}_{m \times n}\right)$ where $m$ is the number of hydro power stations and $n$ is the time interval. Thus the component $x_{i \times j}(i=1,2, \cdots, m ; j=1,2, \cdots, n)$ of the matrix means the outflows from reservoir of the $i^{\text {th }}$ hydro power station in $j^{\text {th }}$ time interval.

\subsection{Problem Formulation}

In this section, the problem formulation model and some theoretical properties of the methods studied in this paper are presented. Aiming to make reading of the remaining of this paper easier, the notations are shown bellow. The notations describe fixed parameters of the small hydroelectric plants, index and variables.

$m$ total of hydro plants;

$K$ set of upstream reservoirs of plant $i$ 
$k$ power plant $\in K$

$i$ index of hydro plants, so that $i=1, \cdots, m$;

$j$ index of time, so that $j=1,2, \cdots, n=24$;

$H_{i, j}$ average head available to $i^{\text {th }}$ hydro plant $[m]$;

$I_{j}$ natural inflow into reservoir at the end of hour $j\left[\mathrm{~m}^{3} / \mathrm{s}\right]$;

$P_{i, j}$ power generation $[k W h]$;

$P_{i, \text { min }}$ minimum power generation of hydro plant $[k W h]$;

$P_{i, \max }$ maximum power generation of hydro plant $[\mathrm{kWh}]$;

$Q_{i, j}$ discharged outflow in the $i^{\text {th }}$ hydro plant $\left[\mathrm{m}^{3} / \mathrm{s}\right]$;

$Q_{i, \text { min }}$ minimum discharge outflow in the $i^{\text {th }}$ hydro plant $\left[\mathrm{m}^{3} / \mathrm{s}\right]$;

$Q_{i, \max }$ maximum discharge outflow in the $i^{\text {th }}$ hydro plant $\left[\mathrm{m}^{3} / \mathrm{s}\right]$;

$q_{i}$ water discharge for multiples uses (downstream) $\left[\mathrm{m}^{3} / \mathrm{s}\right]$;

$S_{i, j}$ water spillage of hydro plant $\left[\mathrm{m}^{3} / \mathrm{s}\right]$;

$V_{i, j}$ water volume of reservoir $i$ at the end of hour $j\left[\mathrm{hm}^{3}\right]$;

$V_{i, \text { min }}$ minimum water volume of reservoir $\left[\mathrm{hm}^{3}\right]$;

$V_{i, \max }$ maximum water volume of reservoir $\left[\mathrm{hm}^{3}\right]$;

$V_{i, \text { begin }}$ initial storage volume of reservoir $\left[\mathrm{hm}^{3}\right]$;

$V_{i, \text { end }}$ final storage volume of reservoir $\left[\mathrm{hm}^{3}\right]$;

$\eta_{i}$ efficiency factor of $i^{\text {th }}$ hydro plant in relative units;

$\triangle t$ time period $[3,600 s]$.

On the short-term economic dispatch of hydro plant problems can be finding out the water releases from each reservoir, that is $\left(Q_{i, 1}, Q_{i, 2}, \cdots, Q_{i, j}\right)$ where $i$ is a certain particle and $j$ is the number of the time period, that maximizes the total hydroelectric generation while meeting various operational and physical constraints. The short-term optimal daily scheduling of hydro plants can be mathematically formulated as a nonlinear optimization problem as follows:

$$
\max F=\sum_{i=1}^{m} \sum_{j=1}^{24} 0.00981 \eta_{i} H_{i, j} Q_{i, j}
$$

Subject to: 
- Hydro plant power generation limits

$$
P_{i, \min } \leq 0.00981 \eta_{i} H_{i, j} Q_{i, j} \leq P_{i, \max }
$$

- Hydro plant outflow limits

$$
Q_{i, \min } \leq Q_{i, j} \leq Q_{i, \max }
$$

- Reservoir storage volumes limits

$$
V_{i, \min } \leq V_{i, j+1} \leq V_{i, \max }
$$

- Initial and terminal reservoir storage volumes

$$
V_{i, 1}=V_{i, \text { begin }} \quad V_{i, 25}=V_{i, \text { end }}
$$

- Water dynamic balance equation for each reservoir

$$
V_{i, j+1}=V_{i, j}-\left(Q_{i, j}+q_{i}+S_{i, j}\right) \triangle t+\sum_{k \in K_{i}}\left(Q_{k, j}+S_{k, j}+I_{j}\right) \triangle t
$$

The water balance equation (18) relates storage and outflow: reservoir storage at end of stage $j$, beginning of stage $j+1$, is equal to initial storage minus outflow volumes (water flow through turbine, spilled flow and water discharge for multiples uses) plus inflow volumes (coming from rain, releases from upstream plants, runoff water and natural river flow). The conversion factor, $\triangle t$, is applied to convert water flow units $\left(\mathrm{m}^{3} / \mathrm{s}\right)$ into water volume units $\left(\mathrm{m}^{3}\right) . K_{i}$ is the set of upstream reservoirs of plant $i$ and in equation (18) is explicitly taken into account the water release of $k$ power plants upstream of plant $i$.

\subsection{Handling Constraints in M-UPSO}

In M-UPSO each particle flying in the search space is considered a potential solution. A set of outflow decision variables, for each hydro plant $\left(Q_{i, j}\right)$, is selected as the position of the particle in each dimension and these values are within the hydro plant outflow bounds given by equation (15). To satisfy other constraints, a penalty function may be applied, so the constrained optimization problem may be transformed into an unconstrained optimization problem, shown in equation (19) as proposed by Yanbin \& Xiaohui (2010).

$$
f=\max \left[F-\left(\gamma_{1} \sum_{i=1}^{m} \sum_{j=1}^{24}\left(V_{i, j+1}-V_{i, \lim }\right)^{2}+\gamma_{2} \sum_{i=1}^{m} \sum_{j=1}^{24}\left(P_{i, j}-P_{i, \lim }\right)^{2}\right)\right]
$$

Where the term $V_{i, \text { lim }}$ and $P_{i, \text { lim }}$ are described in equation $(20) ; \gamma_{1}$ and $\gamma_{2}$ are penalties coefficients.

$V_{i, \lim }=\left\{\begin{array}{ll}V_{i, \max } & \text { if } V_{i, j+1}>V_{i, \max } \\ V_{i, \min } & \text { if } V_{i, j+1}<V_{i, \min } \\ V_{i, j+1} & \text { otherwise }\end{array} \quad P_{i, \lim }= \begin{cases}P_{i, \max } & \text { if } P_{i, j}>P_{i, \max } \\ P_{i, \min } & \text { if } P_{i, j}<P_{i, \min } \\ P_{i, j} & \text { otherwise }\end{cases}\right.$ 


\subsection{M-UPSO Procedure for Optimal Daily Reservoir Operation}

The calculation steps for optimal daily reservoir operation calculation based on M-UPSO algorithm, which is represented as follows:

Step 1. Initialize population following (a) and (b) procedures. Set iteration counter $k=0$;

a. The current position of the $i^{\text {th }}$ particle $\vec{X}_{i}$ is initialized with random real numbers within the range of the decision variable $Q_{i, \max }$ and $Q_{i, \min }$. To generate initial good population, apply for $Q_{1,1}$ to $Q_{n, m}$, the initialization model:

$$
Q_{1 \ldots n, 1 \ldots m}=Q_{i, \min }+\left[\operatorname{rand}(0,1)\left(Q_{i, \max }-Q_{i, \min }\right)\right] .
$$

It is very important to create a population of individuals that satisfy the outflow constraints. Each individual position is represented in each hydro plant, at every dispatch horizon $t$, as a vector $\vec{X}_{i}=\left[Q_{1,1}, \ldots, Q_{1, n}, Q_{2,1}, \ldots, Q_{2, n}, \ldots, Q_{m, 1}, \ldots, Q_{m, n}\right]$;

b. After creating the initial position of each particle, the velocity is also initialized with a uniformly distributed random number in $[0,1]$. The following strategy is used in creating the initial velocity $\vec{V}_{i}=\operatorname{rand}(0,1)$ for $i=1, \ldots, m$.

Step 2. Set $k=k+1$;

Step 3. Repeat the following loop (step through M-UPSO operators) until all particles are evaluated;

a. Evaluate each particle in the population through equations (9) and (10). The global best position $G_{\text {best }}$ is set to equation (9) which represents $\vec{G}_{i}$. The personal best position $P_{\text {best }}$ uses equation (10) for $\vec{L}_{i}$;

b. Calculate the new velocity $\vec{V}_{i}$, based on equation (11) and the new $\vec{X}_{i}$ by using equation (12);

c. Perform the M-UPSO operations for all particles in the iteration.

Step 5. Evaluate each particle in the population through equation (19);

Step 6. Check for termination criteria: if the termination criterion is not satisfied, then go to step 2 , otherwise show the solution set.

To use all the steps mentioned above, the M-UPSO is coded in the software MATLAB ${ }^{\circledR} 7.0$ and is run on a PC 2 GHz Dual-Core, Operational System Windows 7/32 bits and 3 GB RAM.

\subsection{Case Study}

In recent years the number of SHPP inserted into Brazilian Power System has grown rapidly, especially due to incentives and policies that support renewable and energy efficiency. However, currently there are no techniques available for optimization and efficient operation for 
small reservoirs. Due to low efficiency verified after some operation years, especially with nonefficient operational conditions, the Brazilian National Operator System (O.N.S) has applied efforts aiming that the government approves rigid laws for regulating the SHPP projects. These constraints must increase the difficulty to develop new projects in the field of Small Power Plants. These issues have not been on target studies or research and development projects recently.

This work focuses efforts on applying optimization technique with low computational cost aiming to contribute to the efficient operation of the SHPP. The cascade of small reservoir, which was applied the M-UPSO algorithm, has the characteristics showed on the Table 1. The arrangement of power plants into the cascade scheme is showed on Figure 1.

Table 1 - Small Hydro Power Plant parameters.

\begin{tabular}{c|c|c}
\hline Parameters & Power Plant 1 & Power Plant 2 \\
\hline Max. Power Capacity $[\mathrm{kWh}]$ & 24,400 & 12,400 \\
Min. Power Capacity $[\mathrm{kWh}]$ & 7,000 & 3,800 \\
Util Storage Capacity $\left[\mathrm{hm}^{3}\right]$ & 2.05 & 0.70 \\
Initial Storage $\left[\mathrm{hm}^{3}\right]$ & 1.85 & 0.60 \\
Max. Storage Rate per Day $\left[\mathrm{hm}^{3}\right]$ & 0.25 & 0.20 \\
Efficiency Factor $\eta$ & 0.8737 & 0.8766 \\
Average Head $H[\mathrm{~m}]$ & 56 & 25 \\
\hline
\end{tabular}

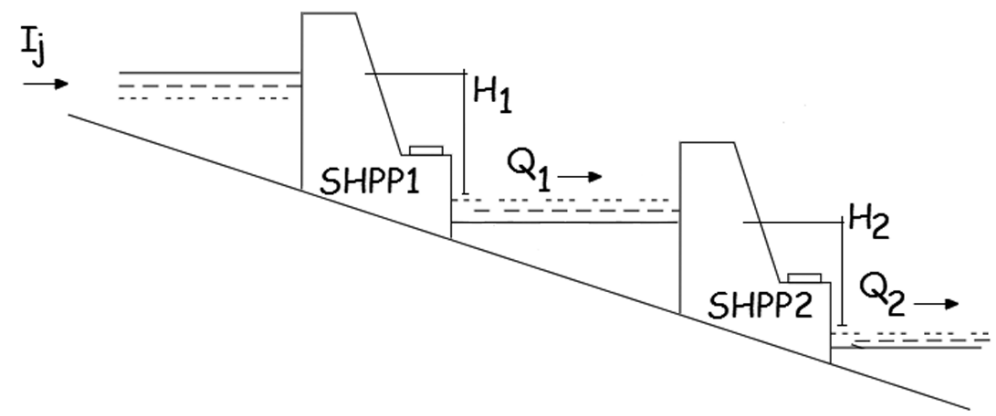

Figure 1 - Cascade of two Small Hydro Power Plants.

This cascade is located on the Pomba river watershed that belongs to basin of the "Rio Paraiba do Sul". The geographic location is in the Southwest Brazil in Minas Gerais region. The inflows have highly seasonal characteristics, which introduce variability on the energy production and require optimization techniques to improve the total production.

To solve the short-term daily scheduling, which targets on five days of operation planning, the M-UPSO algorithm was applied. The results obtained were compared with the U-PSO scheme, standard PSO and Simulated Annealing algorithm. The parameters used in M-UPSO, U-PSO and PSO algorithm are described on Table 2. For the Simulated Annealing algorithm the parameters 
used were: cooling factor 0.9 and maximum iteration allowed 2,300. The stopped criteria for all algorithms used is related to the maximum storage reservoir variation allowed per day.

Table 2 - Parameters of algorithms used to solve DHGS problem.

\begin{tabular}{c|c|c|c}
\hline Parameters & M-UPSO & U-PSO & PSO \\
\hline Max. Iteration Number & 2,300 & 2,300 & 2,300 \\
Swarm Size & 24 & 24 & 24 \\
Inertial Weight $\omega_{\max }$ & 0.90 & not applied & 0.90 \\
Inertial Weight $\omega_{\min }$ & 0.55 & not applied & 0.55 \\
Unification factor $u$ & 0.5 & 0.5 & not applied \\
Constriction Coefficient $\chi$ & 0.729 & 0.729 & not applied \\
\hline
\end{tabular}

The inflows $\left(\mathrm{m}^{3} / \mathrm{s}\right)$ utilized are related to five days, hour-by-hour observed values, starting on May 7 to May 10, 2012, as shown on Figure 2.


Figure 2 - Inflows into SHPP1 reservoir. 
The results for DHGS' problem are reported in Table 3. The two bests results were provided by M-UPSO and UPSO. All constraints were observed and the proposed algorithm M-UPSO could improve the daily production with the same inflows used for others algorithm optimization model.

Table 3 - Results for DHGS problem applying M-UPSO algorithm.

\begin{tabular}{c|c|c|c|c}
\hline $\begin{array}{c}\text { Scheduling } \\
\text { Day }\end{array}$ & $\begin{array}{c}\text { M-UPSO } \\
(\mathrm{kWh})\end{array}$ & $\begin{array}{c}\text { U-PSO } \\
(\mathrm{kWh})\end{array}$ & $\begin{array}{c}\text { PSO } \\
(\mathrm{kWh})\end{array}$ & $\begin{array}{c}\text { Simulated Annealing } \\
(\mathrm{kWh})\end{array}$ \\
\hline $05 / 07 / 2012$ & $513,368.48$ & $513,246.92$ & $512,556.41$ & $512,799.61$ \\
$05 / 08 / 2012$ & $557,083.98$ & $556,961.11$ & $557,028.07$ & $556,916.68$ \\
$05 / 09 / 2012$ & $697,812.72$ & $697,606.32$ & $696,729.40$ & $696,757.53$ \\
$05 / 10 / 2012$ & $602,927.22$ & $602,673.91$ & $602,632.57$ & $602,681.02$ \\
$05 / 11 / 2012$ & $552,073.03$ & $551,803,84$ & $551,961.64$ & $551,767.45$ \\
\hline Total (kWh) & $\mathbf{2 , 9 2 3 , 2 6 5 . 4 4}$ & $\mathbf{2 , 9 2 2 , 2 9 2 . 0 9}$ & $\mathbf{2 , 9 2 0 , 9 0 8 . 0 9}$ & $\mathbf{2 , 9 2 0 , 9 2 2 . 3 0}$ \\
\hline
\end{tabular}

\section{CONCLUSIONS}

In this paper, an optimal operation model for the small hydro power plants is established. The model takes into account capacity and electricity quantity benefits. A modified particle swarm optimization (M-UPSO) algorithm solves the proposed model. The proposed M-UPSO algorithm is easy to implement and does not require additional computational complexity. This algorithm showed quite promising results: the ability to jump out of the local optima is considerably improved, the convergence precision and speed are remarkably enhanced, and thus the high precision and efficiency of the PSO algorithm are achieved.

Furthermore, the solution to the daily scheduling of cascade small of reservoirs, those with the purpose of increasing capacity and power generation benefits, is done by adopting M-UPSO algorithm. Obtaining a good solution for this type of optimization problem is hard, but the results from extensive testing of the proposed M-UPSO compared with the U-PSO, PSO and Simulated Annealing, in this case, confirm the validity of the developed approach.

\section{REFERENCES}

[1] Acosta LC, Revelo JV, Fajardo DF \& Bucheli AP. 2012. Economic dispatch of renewable power generators in a microgrid. Alternative Energies and Energy Quality (SIFAE), 2012 IEEE International Symposium on, pages 1-6.

[2] ANEEL - AgÊncia NaCional de Energia ElÉtriCA. 2003. Critrérios para o enquadramento de aproveitamento hidroelétrico como PCH, Resolução 652 da Agência Nacional de Energia Elétrica - BRASIL, 2003.

[3] Clerc M \& Kennedy J. 2002. The particle swarm, explosion, stability, and convergence in a multidimensional complex space. IEEE Trans. Evol. Comput., 6(1): 58-73. 
[4] Anagnostopoulos JS \& PAPAntonis DE. 2007. Optimal sizing of a run-of-river small hydropower plant Energy Conversion and Management, 48(10): 2663-2670.

[5] Kennedy J \& Eberhart R. 1995. Particle Swarm Optimization. IEEE International Conference on Neural Networks, 27: 1942-1948.

[6] Kennedy J. 2003. Bare bones particle swarms. In Proc. IEEE Swarm Intelligence Symposium, Indianapolis, USA. IEEE Press: pp. 80-87.

[7] Mendes R, Kennedy J \& Neves J. 2004. The fully informed particle swarm: Simpler, maybe better. IEEE Trans. Evol. Comput., 8(3): 204-210.

[8] Parsopoulos KE \& VRahatis MN. 2004. UPSO: A unified particle swarm optimization scheme. Proceedings of the International Conference of Computational Methods in Sciences and Engineering (ICCMSE 2004): VSP International Science Publishers, Zeist, The Netherlands, 1: 868-873.

[9] Peña R, Medina A, Anaya-Lara O \& McDonald JR. 2009. Capacity estimation of a minihydro plant based on time series forecasting. Renewable Energy, 4(5): 1204-1209.

[10] Shi Y \& EBERHART RC. 1998. A modified particle swarm optimizer. IEEE World Congress on Computational Intelligence, IEEE Press: pp. 1951-1957.

[11] Shi Y \& Eberhart RC. 1998. A modified particle swarm optimizer. Proceedings of the IEEE Congress on Evolutionary Computation, NJ: IEEE Press: pp. 303-308.

[12] TRELEA IC. 2003. The particle swarm optimization algorithm: Convergence analysis and parameter selection. Information Processing Letters, 85: 317-325.

[13] Varfolomejeva R, Umbrasko I \& Mahnitko A. 2013. Algorithm of smart control system operation of small hydropower plant. Environment and Electrical Engineering (EEEIC), 12th International Conference on, pp. 330-334.

[14] Yanbin Yuan \& Xiaohui Yuan. 2010. An improved PSO approach to short-term economic dispatch of cascade hydropower plants. Kybernetes, 39(8): 1359-1365. 